\title{
HUMAN-MACHINE INTERACTION IN SOLVING TASKS OF THE PLANNING DEPARTMENT
}

\author{
Boris Kucherov*, Vladimir Artyushenko
}

\author{
Department of information technology and control systems, Information and Technology Faculty, University of \\ Technology, Gagarina 42, Korolev, Moskovskaya oblast, Russia \\ * corresponding author: boris.ku4erov@gmail.com
}

ABSTRACT. The paper discusses issues of human-machine interaction in solving tasks of the planning department under severe resource restrictions using information technology.

The negative factors influencing specialists of the planning department in solving their tasks under the given circumstances are shown. Specific features of designing the user interface in this subject area are noted. Directions to increase the efficiency of reaction of the planning department's specialists to change the current situation by visual and sound notification of various events are marked. Various ways to develop user interface to generate a conflict-free plan under severe resource restrictions are considered. The variants of informative presentation of operational and statistical information to stakeholders are analyzed. These issues are discussed by the example of the planning department which solves the tasks of allocation of control facilities for spacecraft (a subset of satellite range scheduling problem).

KEYWORDS: planning department, human-machine interaction, notification, conflict-free plan, spacecraft.

\section{INTRODUCTION}

The allocation of shared resources (resources of collective usage) is a current problem with a large number of different modifications [15]. Planning departments solving such tasks are used in many industrial areas, such as transportation industry, rocket and space industry, nuclear power industry, etc. As examples, planning departments of railway traffic, planning departments of road works, department of logistics transportation, traffic control departments, etc. [4-9].

In modern world such departments often work under severe resource restrictions caused not only by scarcity of allocated resources, but also by high requirements on time for solving the tasks. These requirements could be satisfied by using modern information technology. However, interaction of specialists with an automated information system of a planning department has sufficient influence on the efficiency of solving the tasks even when there is a high degree of automation.

The main negative factors influencing the specialists of a planning department in solving their tasks under current conditions may include:

- a large amount of data to be processed, a significant number of factors to be analyzed;

- strict requirements to time of solving tasks under conditions when specialists should be resistant to stress;

- a necessity of the current situation continual monitoring for a prompt response to its changes.

The usage of successful approach to human-machine interaction can significantly increase the efficiency of solving tasks of a planning department. The issues of human-machine interaction in the already mentioned subject area are considered by the example of planning department solving tasks of allocation of control facilities for spacecraft. In such case, the control facilities are considered as shared resources (resources of collective usage) and the spacecraft are considered as their consumers. The control facilities (ground stations) are used to carry out communication sessions with spacecraft to receive telemetry information, to transfer command and program data, to measure parameters of spacecraft orbit, etc. [10, 11]. Herewith, a control facility can carry out only one communication session with only one spacecraft at any given time.

The problem of allocation of control facilities for spacecraft can be considered as a subset of satellite range scheduling (SRS) problem. Most papers related to SRS [4, 5, 12, 13, discuss mainly a schedule (plan) optimization. Various approaches, based on an integer linear programming [12, genetic algorithms [13 17, graph color theory [14, 18, etc. [2, 6, 19, 20], are proposed. However, not enough attention is paid to issues of human-machine interaction in solving tasks of allocation control facilities for spacecraft under severe resource restrictions using information technology.

\section{THE INCREASING EFFICIENCY OF RESPONSE OF THE PLANNING DEPARTMENT'S SPECIALISTS ON CHANGING THE CURRENT SITUATION}

One of the main factors, influencing the efficiency of solving tasks of a planning department, is a timeli- 
ness of specialists' response on changing the situation. This factor could be parried by prompt notification of specialists about changing the situation.

Such notification has a number of specificities. First of all, a group of persons to be informed is not so large (from few to tens). Employees' workplaces equipped with computers are located on a quite small area (one or few rooms). The data flows of notifications have address direction. Generally 21, 22, specialists of a planning department are always at their workplaces during their shift (except time for endorsing originals of documents and submitting them for approval), so they can be notified about changes in the current situation by computers.

Receiving information into a database can be considered as a completion of one technological operation and a start of another.

When receiving new information from subscribers, specialists of a planning department are notified about this fact using both visual and sound messages. Its implementation can be based on principles of multimodal alerts used in various services and applications (email, social media, organizers, etc.). After receiving a new message a pop-up window with brief information about it appears and a playback of sound alert or speech comment is played [23+27. The notification may vary depending on the properties of the received information and of the current situation. It enables the specialist to make a decision promptly about the level of urgency to process the received data even if he/she performs other tasks and does not work on the computer at that time. The specialist can interrupt the current task and switch to performing a more priority one or begin processing the received information after the completion of the current task.

The visual component of notification is a pop-up window containing data about the received information. An individual icon can be assigned to each type of event. A specialist can switch to processing the received data directly from the message of visual notification. The sound notifications may vary depending on the properties of the received information. They may be repeated at specified intervals before receiving notification by the specialist.

However, notification about large number of events during short time can cause cognitive overload of specialists of a planning department. Such overload could be prevented by tasks reallocation [28]. For a small number of specialists on the shift (e.g., 2-3 persons) and a large number of simultaneously occurring events, notifications can be arranged by its priorities and processed consistently according to their priorities in addition to tasks reallocation.

During the process of notification of specialists about changes of situation, all information is received into a database, which is a central element of planning department's automated information system. The generalized diagram of propagation of messages about receiving new information is presented in Figure 1

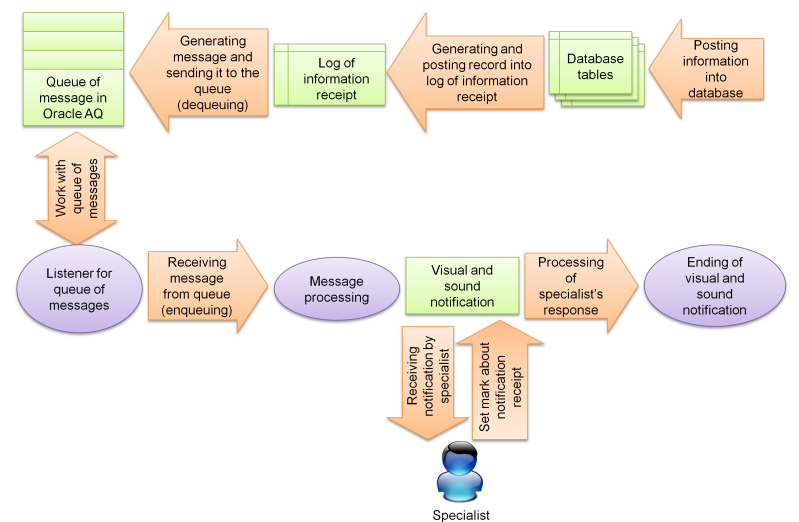

FIGURE 1. The propagation of messages about receiving new data

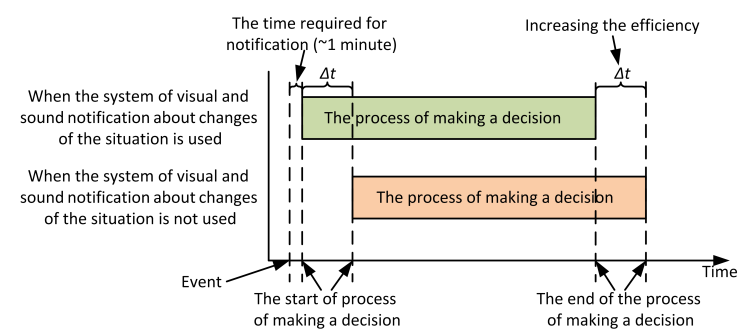

FiguRE 2. Schematic representation of the principle of increasing the efficiency of solving tasks of planning department by using the notification system about changes of situation

22. It was implemented by a mechanism of advanced queues support in database management system Oracle - Oracle Advanced Queuing. The visual component of notifications (message) was implemented by DevExpress VCL (Visual Component Library).

It should be noted that the usage of notification about changes of situation usually does not influence the time of making a decision, but it enables the specialists to start making a decision earlier (see Figure 2). This causes one of two consequences (or their combination): the decision making process is completed earlier or there is more time to make a decision. Thus, the notification about changes of situation can not only increase the efficiency of solving tasks of a planning department by earlier start and, as a consequence, by the earlier end of the decision making process: it can also contribute to improving the decisions due to additional time for its production.

When the notification is received by the specialist and all more priority tasks are completed, then the processing of received data starts. For example, after the planning department receives requests on using control facilities, developing plan of allocation of control facilities starts. Another example can be receiving data about changing the state of control facilities - data preparation for analysis of current situation begins after it.

Processing various data about changing the situation may require different time. So, the processing of 
such data as requests on using control facilities that don't lead to conflict situations (which will be discussed below), request for cancellation of communication sessions, maintenance plans received beforehand, consists of performing a sequences of typical (routine) actions. That is, the processing of such data is carried out at the rule-based level of Skill-Rule-Knowledge framework of Rasmussen [29] and does not create a large cognitive load on specialists. Processing other data such as requests on using control facilities that lead to conflict situations, troubles when performing communication sessions, malfunctions of control facilities is carried out at the knowledge-based level which creates a significant cognitive load on specialists and require more time to process.

\section{Developing a CONFlict-Free PLAN UNDER STRICT RESOURCE RESTRICTIONS}

Issues of human-machine interaction during solving the task of developing conflict-free plan under strict resource restrictions have significant influence on the efficiency and the quality of solving this task. So, information capacity and ergonomic of user interface of software for developing a plan are very important. It is especially significant when the scarcity of shared resources grows due to increasing the number of consumers of shared resources under resource restrictions. Such circumstances cause increasing the number of conflict situations (conflicts). The conflict is a contradiction between request on using shared resources and existing restrictions [21]. It can be caused by the need to use the same resource for different consumers at the same time, the need to use the resource at the time of service operations, etc. Resolution of conflicts is rather time-consuming process. Moreover, it is hard for specialists of planning department to solve successfully a large number of conflicts under severe resource restrictions without using appropriate software. As a result, growing number of conflicts causes the increase of workload of specialists of planning department.

So, developing the user interface of software for planning department should include proactive steps aimed to parry the increase of workload of the specialists. For such purpose it is necessary to predict a situation in the allocation of control facilities for spacecraft. Also, the results of situation prediction can be used to make decisions during developing a plan to control facilities allocation.

\subsection{Prediction WORKLOAd of SPECialists OF PLANNING DEPARTMENT}

To solve tasks of workload prediction of specialists it is necessary to take into account a number of factors: structure and state of shared resources (control facilities), number and properties of requests on its usage, statistical data about its usage, planned maintenance, etc. As it has been already mentioned, one
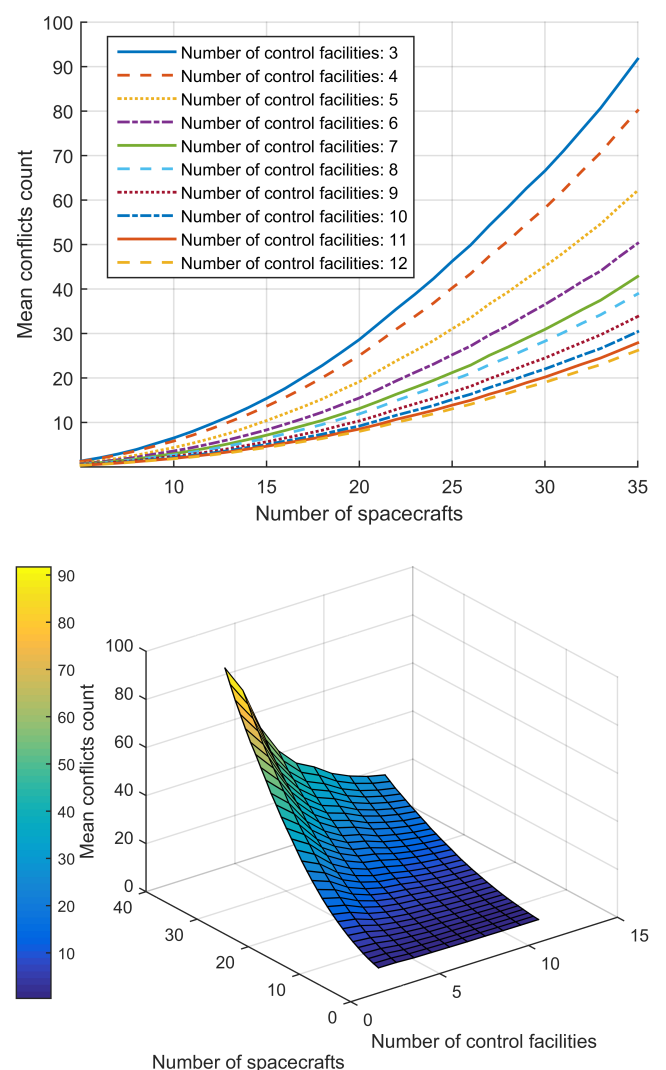

Figure 3. The dependence of a quantity of conflicts per day from the number of spacecrafts and the number of control facilities (calculated in 100 model experiments): 2D-plot (top) and 3D-plot (bottom)

of the major factors influencing the workload of specialists is a number of conflicts, arising during the allocation of control facilities for spacecraft. The number of conflicts significantly depends on the number and properties of requests on the use of control facilities as well as structure and state of control facilities. The number and properties of requests depend on structure and state of spacecrafts constellation and their mission profiles (technological control cycles of spacecrafts).

The workload of specialists of planning department can be predicted by evaluating the number of conflicts which occur when changing the structure and state of spacecraft constellation and control facilities, using series of model experiments. Each series can be used to determine the dependence of each indicator (e.g., number of conflicts) from some factors (e.g., the number of spacecraft).

The example of dependence of quantity of conflicts from the number of consumers for the various number of planning objects is presented in Figure 3

This dependence was obtained as a result of series of 100 model experiments using the model, developed to solve the tasks of workload prediction of specialists of planning department.

The developed model implemented in MatLAB, which allows getting numerical probability characteristics of the situation in allocation of control facilities 
for spacecrafts, contains the following units:

- a unit of loading initial data;

- a unit of calculation windows of radio visibility for spacecrafts by control facilities;

- a unit of generation different sets of input data for series of model experiments;

- a unit of generation requests on using control facilities for spacecrafts (choosing windows of radio visibility to carry out communication sessions);

- a unit of detection and count conflicts;

- a unit of statistical data processing.

The calculation of windows of radio visibility for spacecrafts was implemented using Orekit - a low level space dynamics library written in Java. Precision of Orekit was proved during its usage in real missions 30 .

Windows of radio visibility were calculated using input data in the form of TLE (two-line elements), which are provided by NORAD (North American Aerospace Defense Command) and publicly available for free in its catalog at 31. Sampling 140 spacecraft on low-Earth orbits from this catalog was performed. Windows of radio visibility between these spacecrafts and 4 ground points were calculated. It was mentioned that there are 3 control facilities on each ground point. Time of preparation for communication sessions was set as 15 minutes.

A hypothetical constellation of 35 spacecrafts was generated during each experiment by random sampling spacecrafts from the previously sampled ones. For each constellation requests on using control facilities were generated from various number of spacecrafts (from 5 to 35 ) for various number of control facilities (from 3 to 12 facilities on 4 ground point). The requests were generated using approach of random sampling 3 windows of radio visibility from the most preferred 10 ones (which have the largest duration). Conflicts were detected and counted for each subset of requests.

The model results show numerical probability values of progressive increase in number of conflicts during increasing the number of spacecrafts and, consequently, progressive increase of workload of specialists of planning department. The largest number of conflicts is observed at the least number of control facilities. Note that the number of conflicts can vary significantly for the same number of spacecrafts and control facilities (e.g., Figures 4 and 5 .

However, the trend of progressive growth of the number of conflicts during increasing the number of spacecrafts is still preserved. It should be taken into account in developing the user interface of software for planning department.

This is particularly important due to the fact that increasing the number of specialists on the shift will not completely parry the increasing load. It is caused

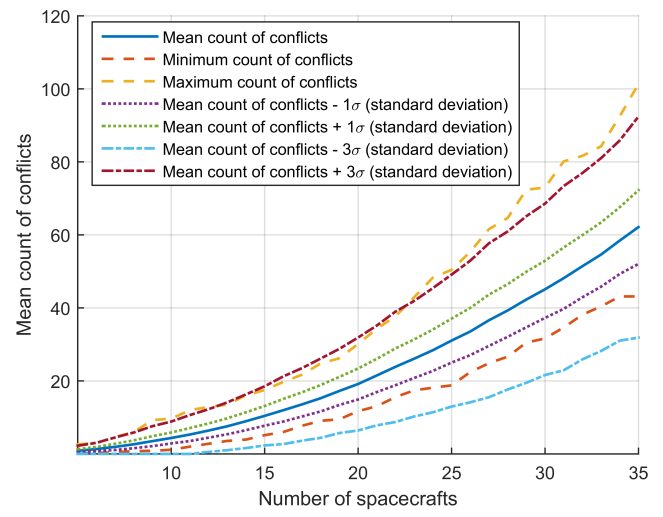

Figure 4. The example of range of variation of the conflicts' number for 5 control facilities

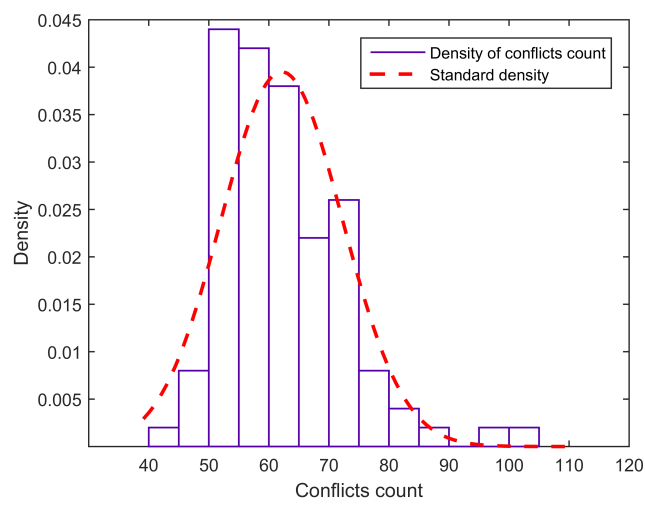

Figure 5. The example of the probability density of the number of conflicts for 35 spacecrafts and 5 control facilities

by the specificities of the task of developing (changing) a plan of allocation of control facilities which do not allow to parallelize its execution between several specialists. For example, parallel solution of several conflicts at the same date by different specialists in the most cases is impractical due to the fact that new conflicts may occur. Furthermore, as shown in [32, for a large number of specialists tasks reallocation between them may lead to a significant increase of resource costs of these operations.

\subsection{The USER INTERFACE FOR DEVELOPING A CONFLICT-FREE PLAN UNDER STRICT RESOURCE RESTRICTIONS}

After receiving requests to use control facilities, they are aggregated to one table. This table is a basis for developing a draft of the plan of allocation of control facilities. The draft (candidate) plan can be viewed in a tabular form (e.g., Figure 6) as well as in a form of a diagram for user convenience. Color indication of conflicts with the possibility to view data about their reasons was implemented. So, the user can view requests that conflict with each other and data about the state of the shared resources which cannot be used in accordance with the requirements of the received requests. 


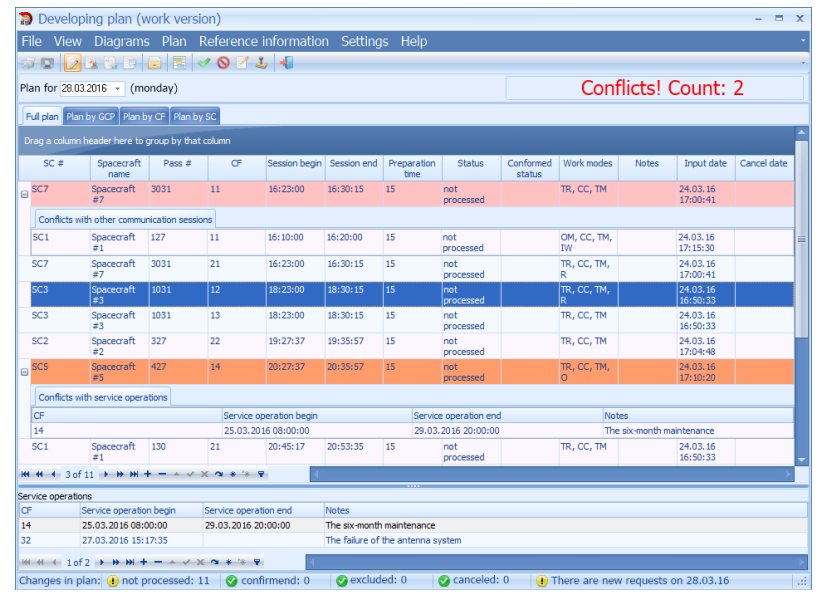

FiguRE 6. The main form of the program for developing conflict-free plan

When new requests on using resources are received, the indication about existing not-processed data is carried out by color highlighting these data and showing total number of records to be processed. Colors used for highlighting not-processed data as well as conflicts are quite important for information perception by the specialists of planning department. On the one hand, colors for alarms (e.g., conflicts) should attract attention to the highlighted data. On the other hand, color indication can be shown during quite long time, so it shouldn't be annoying or cause fatigue of the specialists. Also, various colors should be used for conflicts caused by different reasons (e.g., conflict with another communication session and conflict with maintenance of control facility). As the result of practical use of the developed software, colors with not very high saturation were chosen (hues of red for alarms, hues of blue for not-processed data).

The specialist can resolve conflicts in manual mode as well as in automated mode (using special software for conflicts resolution), or using both of them. He/she chooses appropriate mode after analysing conflicts and reasons of their formation.

Advisability of solving conflicts in manual mode in some situations could be caused by small number of conflicts or by simplicity of solution (e.g., moving a communication session to another control facility without changing its time). Time costs of solving conflicts in manual and automated modes can be comparable in such case.

The resolution of conflicts in typical situations where the decisions are already known and stored in the corresponding database should also be mentioned.

Candidate solutions of conflicts can be developed using visualization of plan in the diagram containing available times of using each resource for each consumer. Such times can be considered as possible solutions of the conflicts. Also, a user can view data about possible solutions of the conflicts. Such view of data representation is more intuitive and can make user's work easier.
However, it makes sense to use software for automated conflicts resolution (developing conflict-free plans) in many cases. A possibility to use such external (third-party) software is provided to ensure resolving conflicts in automated mode. In such case, all necessary data, including a draft of the plan of allocation control facilities, data about existing resource restrictions and reference data- is sent to software for automated development of a conflict-free plan. Note that the specialist could adjust the settings of developing conflict-free plans. For example, the specialist can mark certain communication sessions as mandatory to be included in the plan. Also, the specialist can resolve some conflicts in manual mode before sending a draft of the plan to the software for automated development of a conflict-free plan. In addition, the specialist can make changes in the draft of the plan generated by such software.

When a conflict-free plan is developed, it is sent into the reverse direction (from software for automated developing conflict-free plan to developed software for allocation of control facilities for spacecrafts).

Automated conflicts resolution can be carried out using, for example, methods of solving SRS discussed in 4, 5, 12, 18.

When all conflicts were resolved (if they have arisen), the plan of allocation of control facilities is approved. As soon as it was done, an extract from the plan for each subscriber is generated and sent.

It should be noted that specialists of planning department have to prepare a large number of documents in hard copy after developing or changing the plan. The batch printing of documents on the results of a developing plan is provided to reduce time of this operation. The list of the documents to be printed is dynamically generated according to the content of the plan or its correction.

To develop a conflict-free plan of allocation of control facilities as well as to solve other tasks of planning department it is necessary to have various data about the current situation (e.g., data about state of control facilities). Its perception by the specialists can influence on the efficiency and quality of their decisions. So, its representation is also one of important issues of human-machine interaction in solving tasks of the planning department.

\section{ThE VARIANTS OF INFORMATIVE REPRESENTATION OF OPERATIVE AND STATISTICAL DATA}

Specialists of planning department need compact and informative representation of data about the current situation as well as the results of analysis of using shared resources to perform prompt evaluation and analysis of it. Data about current situation include such parameters as current state of control facilities and spacecrafts, requirements of spacecrafts, and can 
be considered as operative data. The results of analysis of using control facilities for spacecrafts include such data as a number of planned and performed communication sessions, a number of troubles when communication sessions take place and its effects, statistical characteristics of repairing control facilities with typical malfunctions, etc. One of the most informative and convenient for the users ways to represent operative data is to use interactive schematic form and to represent the results of analysis of using control facilities is to use statistics.

\subsection{Representation of operative Data}

Compact and informative representation of operative data can be reached by presenting data in an interactive schematic form with the possibility to drill down to the desired level (e.g., Figure 7).

In this example pictograms of shared resources (control facilities) are schematically shown on geographical map in accordance with their locations. Color indication of the status of control facilities for spacecrafts is carried out both in terms of serviceability and in terms of current usage. Detailed reference and operational data can be viewed for each shared resource.

Also, the pictograms of consumers (spacecrafts) are shown at the top part of the interactive scheme. The indication of status and existence of current communication sessions for spacecrafts is implemented. Detailed reference and operational data can be viewed for each consumer. Also, a set of allowed shared resources is shown for each consumer (a set of control facilities which are included in ground control complex of each spacecraft is shown).

The progress of performing the plan is showed in tabular form for each control facility at the top of interactive scheme. Moreover, a separated tabular form to visualize the current progress of performing the plan of control facilities allocation for spacecrafts with color indication of state of communication sessions is provided.

In addition, the data about the state of spacecrafts constellation are displayed using 3D-model of spacecrafts flights on near-Earth orbit (e.g., Figure 8).

Space dynamics calculations in the developed model are performed using SGP4 model (SGP - Simplified General Perturbations) for spacecrafts on low-Earth orbit and SDP4 model (SDP - Simplified Deep Space Perturbations) for other spacecrafts.

The possibility to manage progress of simulated time is provided. It can be normal or accelerated. Also, a progress of simulated time can be stopped (it would be mean showing the results of modelling at fixed time).

It should be noted that one of the directions of further development is integration between a 3D-model and interactive geographical map. It will have positive influence on the efficiency of evaluating the current situation.

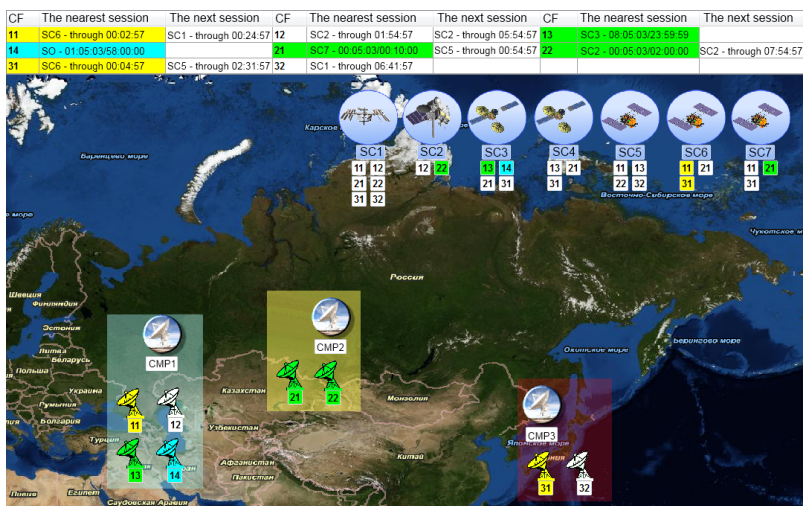

FigURE 7 . The operative presentation data about the current situation in the schematic form $(\mathrm{CF}$ control facility, SC - spacecraft, CMP - control and measurement point)

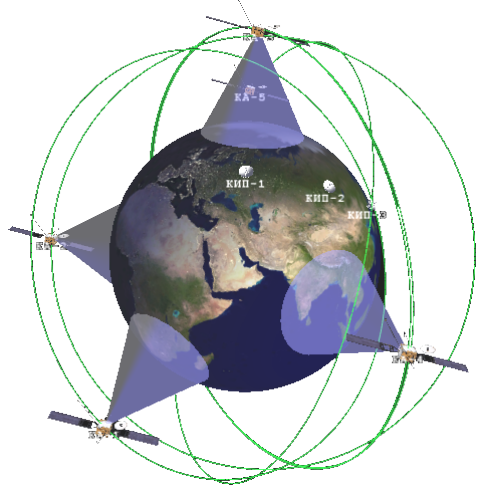

Figure 8. Example of visualization of data about state of spacecrafts constellation

It is important to note that data about current situation is obtained as a result of collecting and processing a large amount of data, such as results of communication sessions and reprimands to them, data about occurrence and parrying malfunctions of control facilities, maintenance of control facilities, etc. Much of this data, e.g. reprimands to communication sessions, can occur many times. So, using a list of typical reprimands is proposed. It favors classification data and improvement of quality of processing data by standardization of definitions. Moreover, it allows increasing the efficiency of input data about reprimands by choosing its description from the list instead of manual input. Possibility to view collected data in summary table for selected time interval was implemented to use in creating reports and further analysis of the data. The data in such table can be filtered and grouped flexibly to concentrate user's attention on a part of the data which should be processed at the present.

\subsection{Representation of Statistical data}

As it was mentioned above, the allocation of shared resources should take into account the results of analysis of their usage. Specialists of planning department need various statistical data to perform such analysis. 
Statistical data manipulation can be done for various periods and various data slices (e.g., consumers, resources, results of resources usage, time intervals, etc.). The flexible generation of reports in accordance with current requirements of users is provided. In addition, there should be an opportunity to calculate new statistical indicators based on the basic ones with applying to them predefined functions and groups.

Statistical data manipulation is implemented both on server-side using features of Oracle Database and on client-side. Server-side implementation is done using aggregate and analytical SQL (Structured Query Language) functions. Client-side implementation is done using components QuantumGrid and PivotGrid from DevExpress VCL. Use of such components provides flexible filtering and grouping statistical information. It helps to increase efficiency of analysis of using control facilities. Also, a user can view data in the standard as well as in a customizable view while working with pivot tables, which are implemented by PivotGrid component. It should be noted that this current view of pivot table can be printed. Flexibility of data representation in pivot tables, achieved by changing their view through moving headers of fields into different table areas, allows to generate reports promptly according to actual requirements of the specialists of the planning department. Data from these tables can also be presented in charts. It ensures increasing not only clearness of data visualization, but also clearness of reports prepared by the specialists of the planning department.

\section{Conclusions}

Thus, the analysis of specific features of humanmachine interaction in solving the tasks of planning department under strict resource restrictions using advanced information technology has been performed. The proposed approaches to developing user interface provide concentrated attention of specialists of planning department on the most important data requiring priority processing. The system of visual and sound notification about important events was developed to increase the efficiency of specialists' response on changes of the current situation. Specific features of user interface for developing conflict-free plan have been noted. The variants of informative presentation of actual information about the current situation on the interactive scheme and flexible presentation of statistical data have been proposed.

\section{REFERENCES}

[1] J. Fliege, K. Kaparis, B. Khosravi. Operations research in the space industry. European Journal of Operational Research 217(2):233 - 240, 2012. DOI:http://dx.doi.org/10.1016/j.ejor.2011.06.035

[2] T. Franco, F. Moreira, S. Hall, H. Oliveira. The Contact Scheduling Process of the Galileo Navigation System, pp. 446-455. Springer Berlin Heidelberg, Berlin, Heidelberg, 2008. DOI:10.1007/978-3-540-69052-8_47.
[3] S. K. Jena, B. Majhi, T. Bakshi, et al. An evolutionary algorithm for multi-criteria resource constrained project scheduling problem based on pso. Procedia Technology 6:231 - 238, 2012. DOI:http://dx.doi.org/10.1016/j.protcy.2012.10.028.

[4] A. J. Vazquez, R. S. Erwin. On the tractability of satellite range scheduling. Optimization Letters 9(2):311-327, 2015. DOI:10.1007/s11590-014-0744-8.

[5] L. Barbulescu, A. Howe, D. Whitley. \{AFSCN\} scheduling: How the problem and solution have evolved. Mathematical and Computer Modelling 43(9-10):1023 1037, 2006. Optimization and Control for Military Applications, DOI:http://dx.doi.org/10.1016/j.mcm.2005.12.004

[6] Z. Zhang, N. Zhang, Z. Feng. Multi-satellite control resource scheduling based on ant colony optimization. Expert Systems with Applications 41(6):2816 - 2823, 2014. DOI:http://dx.doi.org/10.1016/j.eswa.2013.10.014

[7] D. Rozhdestvenskiy, P. Bouchner, A. Mashko, et al. Dynamic human-machine interface for electrical vehicle design guidelines. In Smart Cities Symposium Prague (SCSP), 2015, pp. 1-5. 2015. DOI:10.1109/SCSP.2015.7181573.

[8] O. Pribyl, T. Horak. Individual perception of smart city strategies. In Smart Cities Symposium Prague (SCSP), 2015, pp. 1-6. 2015. DOI:10.1109/SCSP.2015.7181550

[9] J. L. Espinosa-Aranda, R. GarcÃya-RÃşdenas, M. del Carmen RamÃyrez-Flores, et al. High-speed railway scheduling based on user preferences. European Journal of Operational Research 246(3):772 - 786, 2015. DOI:http://dx.doi.org/10.1016/j.ejor.2015.05.052

[10] M. Schmidt, K. Schilling. Ground Station Networks for Distributed Satellite Systems, pp. 355-371. Springer New York, New York, NY, 2013. DOI:10.1007/978-1-4614-4541-8_11

[11] S. Madry, J. N. Pelton, S. Camacho-Lara. Ground Systems for Satellite Application Systems for Navigation, Remote Sensing, and Meteorology, pp. 1095-1110. Springer New York, New York, NY, 2013. DOI:10.1007/978-1-4419-7671-0_11.

[12] R. Vazquez, F. Perea, J. G. Vioque. Resolution of an antenna-satellite assignment problem by means of integer linear programming. Aerospace Science and Technology 39:567 - 574, 2014. DOI:http://dx.doi.org/10.1016/j.ast.2014.06.002

[13] Y. Li, R. Wang, Y. Liu, M. Xu. Satellite range scheduling with the priority constraint: An improved genetic algorithm using a station $\{$ ID $\}$ encoding method. Chinese Journal of Aeronautics 28(3):789 - 803, 2015. DOI:http://dx.doi.org/10.1016/j.cja.2015.04.012

[14] A. Sarkheyli, A. Bagheri, B. Ghorbani-Vaghei, R. Askari-Moghadam. Using an effective tabu search in interactive resources scheduling problem for $\{\mathrm{LEO}\}$ satellites missions. Aerospace Science and Technology 29(1):287 - 295, 2013. DOI:http://dx.doi.org/10.1016/j.ast.2013.04.001

[15] F. Xhafa, X. Herrero, A. Barolli, et al. Evaluation of struggle strategy in genetic algorithms for ground stations scheduling problem. Journal of Computer and System Sciences 79(7):1086 - 1100, 2013. DOI:http://dx.doi.org/10.1016/j.jcss.2013.01.023. 
[16] T. P. Bagchi. Near optimal ground support in multispacecraft missions: A ga model and its results. IEEE Transactions on Aerospace and Electronic Systems 45(3):950-964, 2009. DOI:10.1109/TAES.2009.5259176

[17] J. Lee, S. Wang, D. Chung, et al. Visibility conflict resolution for multiple antennae and multi-satellites via genetic algorithm. In Aerospace Conference, 2013 IEEE, pp. 1-10. 2013. DOI:10.1109/AERO.2013.6496849

[18] N. Zufferey, P. Amstutz, P. Giaccari. Graph colouring approaches for a satellite range scheduling problem. Journal of Scheduling 11(4):263-277, 2008. DOI:10.1007/s10951-008-0066-8

[19] G. Corrao, R. Falone, E. Gambi, S. Spinsante. Ground station activity planning through a multi-algorithm optimisation approach. In 2012 IEEE First AESS European Conference on Satellite Telecommunications (ESTEL), pp. 1-6. 2012. DOI:10.1109/ESTEL.2012.6400125

[20] A. J. Vazquez, R. S. Erwin. Robust fixed interval satellite range scheduling. In 2015 IEEE Aerospace Conference, pp. 1-6. 2015. DOI:10.1109/AERO.2015.7119277

[21] V. Artuschenko, B. Kucherov. Informatization of control for spacecraft constellation. Applied informatics 6(48): 6 - 14, 2013

[22] B. Kucherov. Visual and sound notification program for spacecraft constellation control system. Applied informatics 5(53):25 - 32, 2014.

[23] S. T. Iqbal, E. Horvitz. Notifications and awareness: A field study of alert usage and preferences. In Proceedings of the 2010 ACM Conference on Computer Supported Cooperative Work, CSCW '10, pp. 27-30. ACM, New York, NY, USA, 2010. DOI:10.1145/1718918.1718926

[24] H.-R. Pfister, S. WollstÃd'dter, C. Peter. Affective responses to system messages in human-computer-interaction: Effects of modality and message type. Interacting with Computers 23(4):372 383, 2011. Cognitive Ergonomics for Situated

Human-Automation Collaboration, DOI:http://dx.doi.org/10.1016/j.intcom.2011.05.006

[25] A. Serenko. A model of user adoption of interface agents for email notification. Interacting with Computers 20(4-5):461 - 472, 2008. DOI:http://dx.doi.org/10.1016/j.intcom.2008.04.004

[26] C. L. Paul, A. Komlodi, W. Lutters. Interruptive notifications in support of task management. International Journal of Human-Computer Studies 79:20 - 34, 2015. Integrating Knowledge of Multitasking and Interruptions Across Different Perspectives and Research Methods, DOI:http://dx.doi.org/10.1016/j.ijhcs.2015.02.001

[27] A. J. B. Brush, P. Johns. Speechtoast: Augmenting notifications with speech input focus. In Proceedings of the International Working Conference on Advanced Visual Interfaces, AVI '12, pp. 270-273. ACM, New York, NY, USA, 2012. DOI:10.1145/2254556.2254607

[28] M. Neerincx, J. Veltman, M. Grootjen, J. v. Veenendaal. A model for cognitive task load prediction: Validation and application. In Proceedings of the 15th Triennial Congress of the International Ergonomics Association. Seoul, Korea: IEA2003. 2003.

[29] J. Rasmussen. Information Processing and Human-Machine Interaction: An Approach to Cognitive Engineering. Elsevier, Amsterdam, Netherlands, 1986.

[30] Orekit. www.orekit.org

[31] Norad two-line element sets current data. http://celestrak.com/NORAD/elements/

[32] T. de Jong. Cognitive load theory, educational research, and instructional design: some food for thought. Instructional Science 38(2):105-134, 2010. DOI:10.1007/s11251-009-9110-0 